\title{
A Deep-Learning Hybrid-Predictive-Modeling Approach for Estimating Evapotranspiration and Ecosystem Respiration
}

Jiancong Chen ${ }^{1}$, Baptiste Dafflon ${ }^{2}$, Anh Phuong Tran ${ }^{2,3}$, Nicola Falco ${ }^{2}$, and Susan S. Hubbard ${ }^{2}$

${ }^{1}$ Department of Civil and Environmental Engineering, University of California, Berkeley, CA, USA, ${ }^{2}$ Earth and Environmental Sciences Area, Lawrence Berkeley National Laboratory, Berkeley, CA, USA, ${ }^{3}$ Depatment of Water Resources Engineering and Technology, Water Resources Institute, 8, Phao Dai Lang, Dong Da, Hanoi, Vietnam

Supplementary Materials

1. Deep Learning Model Configuration

Table S.1 Configuration of Deep-Learning Module

\begin{tabular}{cccc}
\hline Layer & Output Shape & Parameters \# & Note \\
\hline LSTM & {$[50,1]$} & 11600 & \\
LSTM & {$[25]$} & 7600 & Rate $=0.1$ \\
Dropout & {$[25]$} & 0 & L2 regularizers, 0.01 \\
Dense & {$[8]$} & 208 & Rate $=0.1$ \\
Dropout & {$[8]$} & 0 & Output Layer \\
Dense & {$[1]$} & 9 & \\
\hline
\end{tabular}

2. HPM and MOD16A2 ET Comparison at East River Watershed

\section{ET estimation from HPM and MODIS at DF1}

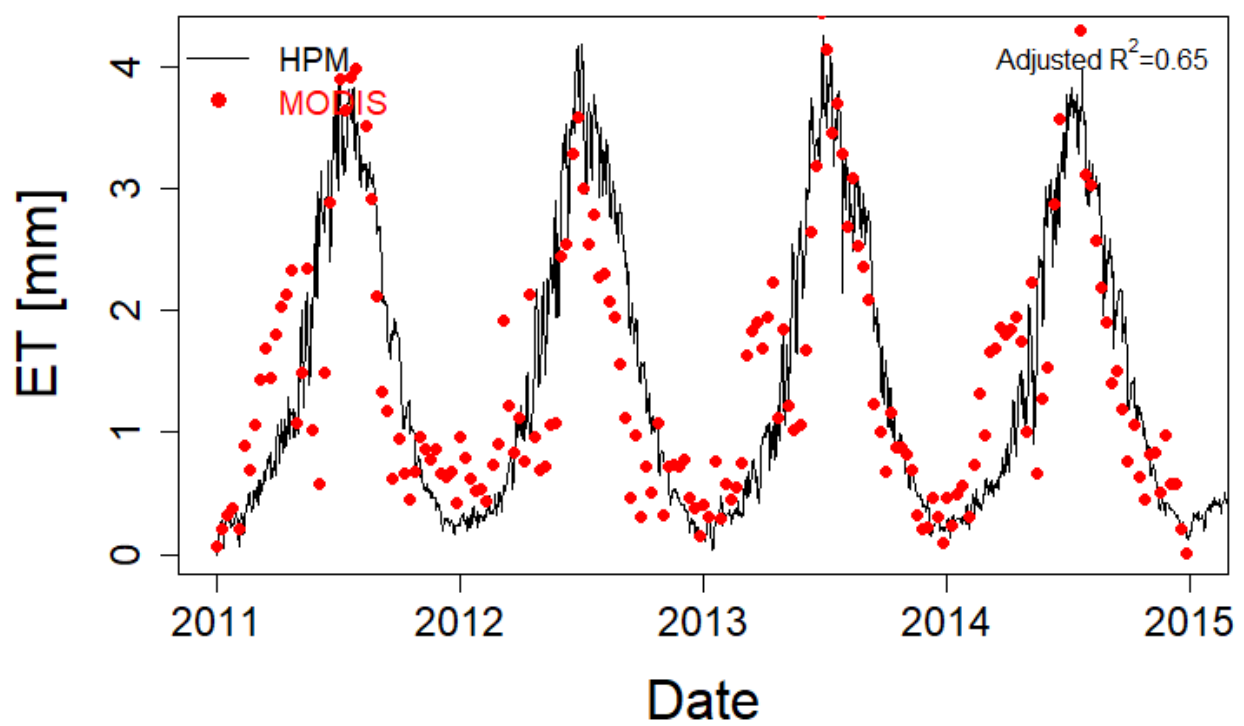

Figure S1. Comparison of 8-day averaged ET estimation from HPM and Mu et al. (2013) at deciduous forests site in East River Watershed.

3. CLM performance at US-NR1 


\section{ET comparison at US-NR1}

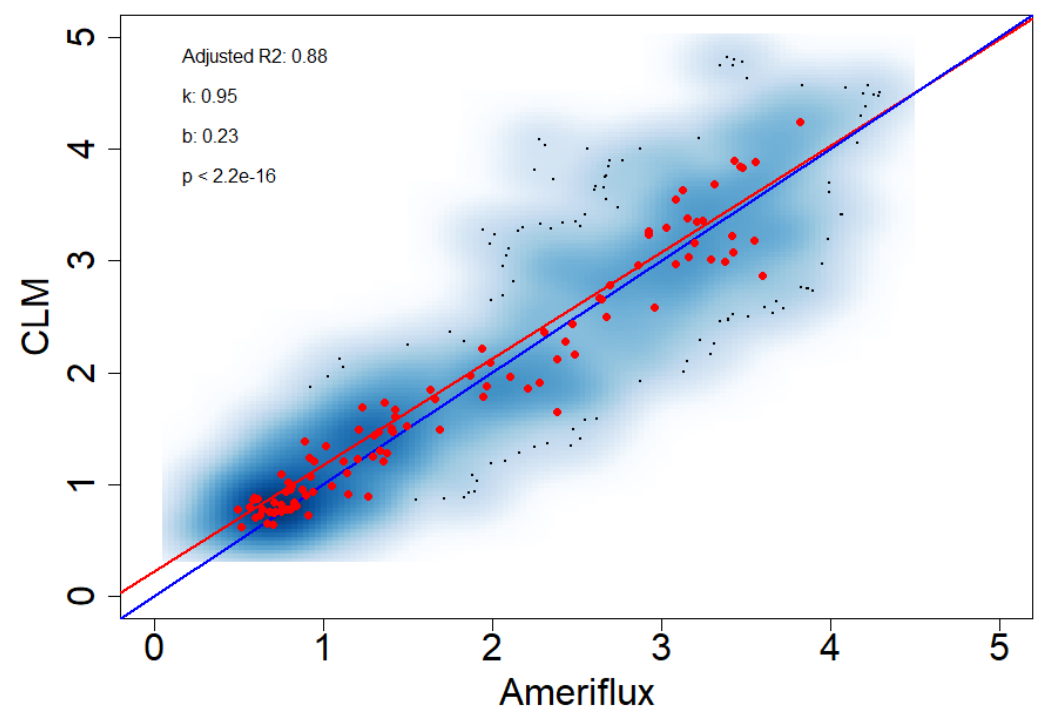

Figure S2. Comparison of ET estimation from CLM and flux tower measurements at US-NR1. Consistency between CLM estimation and direct measurement from flux tower is observed.

4. Meteorological forcings heterogeneity within East River Watershed and across SNOTEL stations 

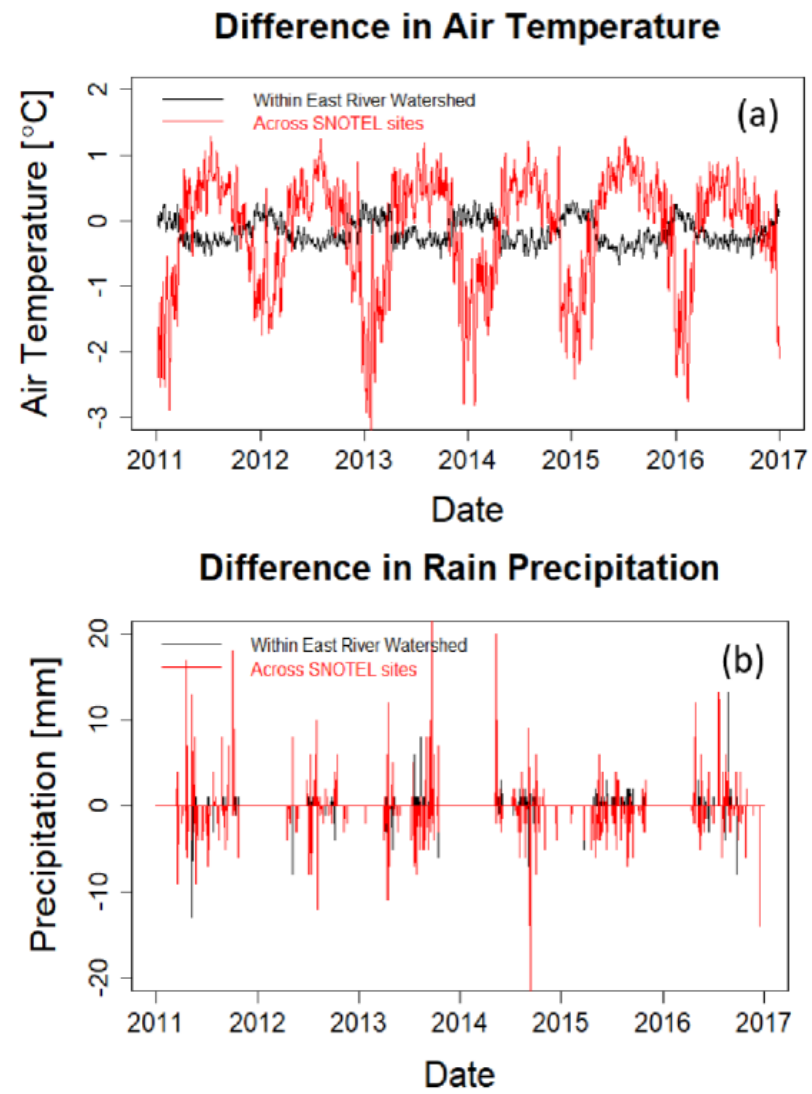

Difference in incident Solar Radiation

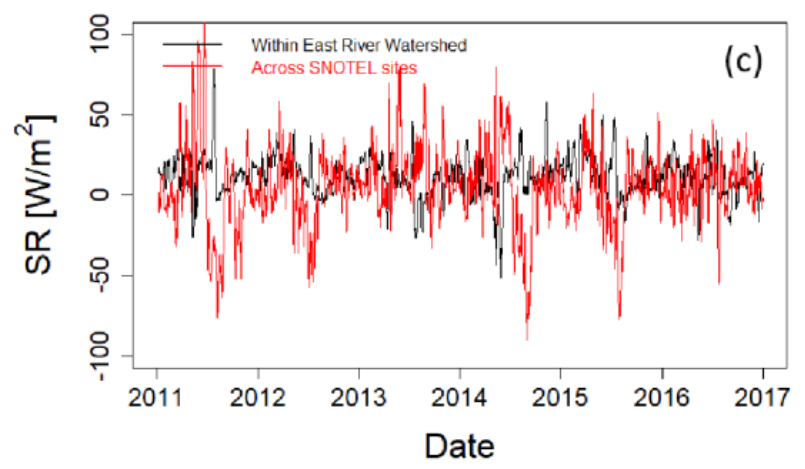

Figure S3. Meteorological forcings heterogeneity within East River Watersheds (DF1 and EF1, black lines) with DAYMET data and across SNOTEL stations (ER-BT and ER-PK, red lines) with SNOTEL data. 

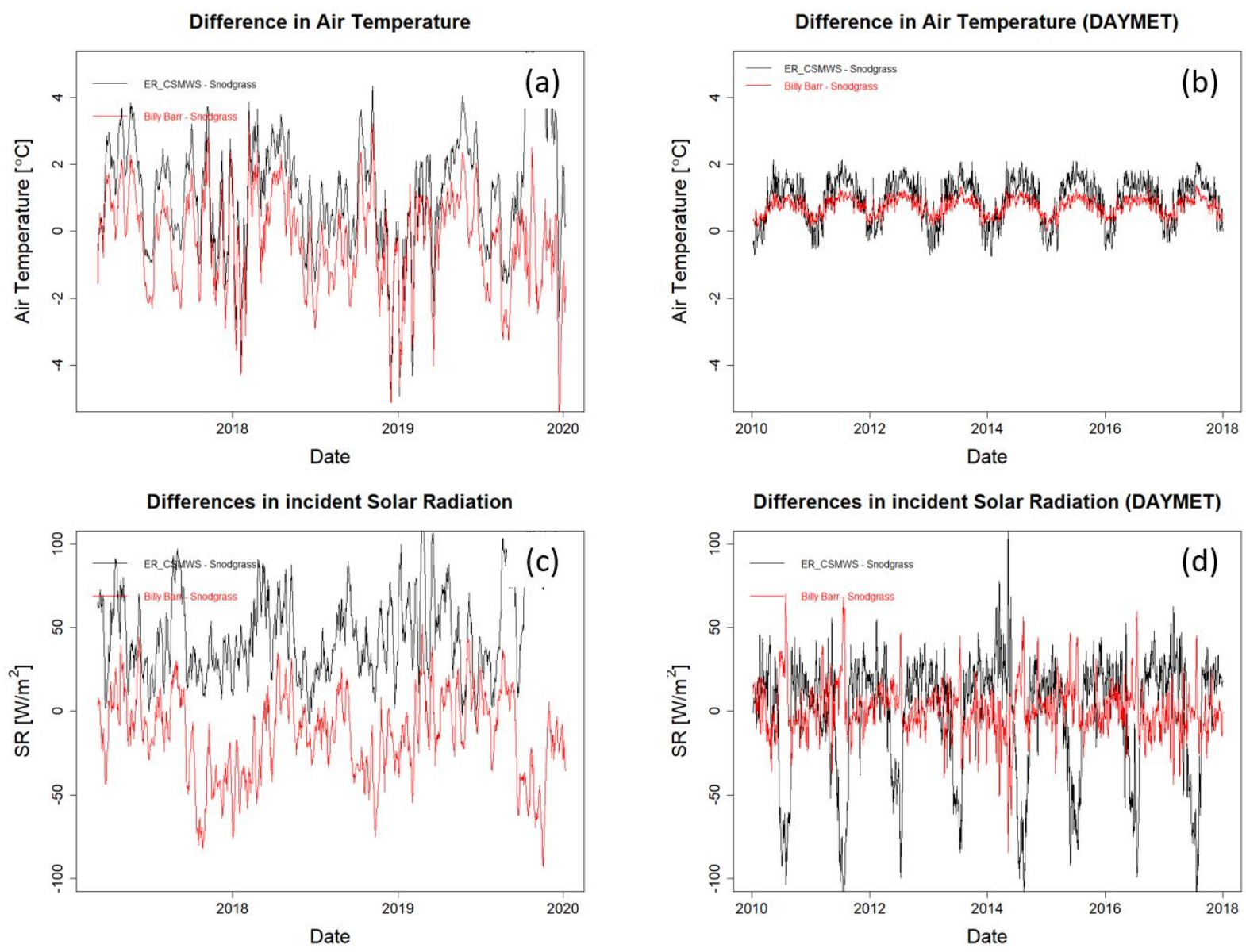

Figure S4. Differences in air temperature and incident solar radiation among three weather stations (ER_CSMWS, Snodgrass and Billy Barr) locations within the East River Watershed. Panel (a) and (c) present data from weather stations obtained from https://wfsfa-data.lbl.gov/. Panel (b) and (d) present data obtained from DAYMET. 